\title{
Self-dual singularity through lasing and antilasing in thin elastic plates
}

\author{
M. Farhat $\odot,{ }^{1, *}$ P.-Y. Chen, ${ }^{2}$ S. Guenneau $\odot,{ }^{3}$ and $\mathrm{Y}$. $\mathrm{Wu}^{1, \dagger}$ \\ ${ }^{1}$ Computer, Electrical, and Mathematical Science and Engineering (CEMSE) Division, \\ King Abdullah University of Science and Technology (KAUST), Thuwal 23955-6900, Saudi Arabia \\ ${ }^{2}$ Department of Electrical and Computer Engineering, University of Illinois at Chicago, Chicago, Illinois 60607, USA \\ ${ }^{3}$ UMI 2004 Abraham de Moivre-CNRS, Imperial College London, London SW7 2AZ, United Kingdom
}

(Received 4 August 2020; revised 9 February 2021; accepted 18 March 2021; published 8 April 2021)

\begin{abstract}
We show here that a coherent perfect absorber and laser (CPAL) enabled by parity-time $(\mathcal{P} \mathcal{T})$-symmetry breaking may be exploited to build monochromatic amplifying devices for flexural waves. The fourth-order partial differential equation governing the propagation of flexural waves in thin-elastic piezoelectric plates leads to $4 \times 4$ transfer matrices, and captures the interplay between propagating and evanescent waves that is translated into $\mathcal{P} \mathcal{T}$-symmetry properties specific to elastic plate systems. We thus demonstrate the possibility of using CPAL for such systems and we argue the possibility of using this concept to detect extremely small-scale vibration perturbation with important outcomes in surface science (imaging of nanometer vibration) and geophysics (improving seismic sensors like velocimeters). The device can also generate finite signals using very low exciting intensities and/or alternatively can be used as a perfect absorber for flexural energy, by tailoring the left and right incident wave amplitude and phase, with evident energy harvesting applications.
\end{abstract}

DOI: 10.1103/PhysRevB.103.134101

\section{INTRODUCTION}

In recent years the use of resonant elements enriched the properties of periodic media with the paradigm shift of metamaterials. These are constructed from a judicious arrangement of physical resonators whose size is very small compared to the typical wavelength of interest [1] and permit some exotic applications such as negative refraction [2] and scattering cancellation technique $[3,4]$. Recently, the concepts of electromagnetic and acoustic metamaterials and metasurfaces [2-4] have been extended to elastic waves in solid structures [5] that possess unique features. For instance, the tensorial nature of the equations governing elastic waves requires complex analytical and numerical modeling that takes into account the coupling between pressure and shear waves at solid interfaces [6]. In the same vein, a particular type of elastic solid, the thin-elastic plate (TEP) has drawn a growing interest in the wave physics community [6,7]. The plate has a small vertical dimension (thickness) in comparison to its lateral dimensions and wavelengths [6], resulting in the vertical displacement of the plate largely determined by the flexural mode (i.e., no shear), sometimes designed as $A_{0}$ mode [6]. The bending of these TEPs can be described efficiently by the Kirchhoff-Love (KL) equation [a fourth-order partial differential equation (PDE)] and interestingly has a scalar nature in the case of isotropic plates [6] [see Supplemental Material (SM) [8] for derivation and model validation]. This feature allows for a more straightforward numerical modeling of waves propagating in isotropic TEPs. Subsequently, several designs have been proposed for flexural waves, including cloaking [9-11],

\footnotetext{
*mohamed.farhat@kaust.edu.sa

†ying.wu@kaust.edu.sa
}

negative refraction [12], localized surface plate modes [13], and elastic plate crystals $[14,15]$.

In a different field of physics, it was shown in 1998 that non-Hermitian Hamiltonians with parity-time $(\mathcal{P} \mathcal{T})$ symmetry possess real eigenvalues [16]. First used in quantum mechanics [17], this property was subsequently applied to photonics because the paraxial wave equation is mathematically equivalent to the Schrödinger equation $[18,19]$, leading to some remarkable properties such as an asymmetric propagation of the modes or the existence of an exceptional point (EP) where the $\mathcal{P} \mathcal{T}$-symmetry is broken $[20,21]$. $\mathcal{P} \mathcal{T}$-symmetry gained a tremendous momentum among the photonics community due to its promising applications, e.g., in environmental sensing [22], optical isolation [23], on-chip optical systems [24], and cavity-mode selection in microring lasers [25]. Recently, the similar asymmetric propagation behavior was reported for acoustic waves in balanced loss and gain layers [26,27]. Intriguing applications of $\mathcal{P} \mathcal{T}$-symmetric acoustic materials have been further envisioned, e.g., in unidirectional invisibility cloaking [28], invisible acoustic sensing [29], phononic lasing [30], and acoustic Willis coupling [31]. For elastodynamic waves, shunted piezoelectric (lead zirconium titanate, i.e., PZT) thin materials may lead to gain/loss in elastic plates (see SM [8]), depending on the resistance of the shunted circuit [32-34], enabling a feasible platform to study $\mathcal{P} \mathcal{T}$-symmetric-based physical properties [35-37].

We show in this paper the possibility to realize the equivalent of lasing in elastic plates, i.e., FLASER, through coherent perfect absorption and lasing (CPAL) effect thanks to gain and loss values of Young's modulus corresponding to the lasing threshold displaying a quantized behavior, which occurs due to the topological character of the system. Both gain and loss are obtained by using piezoelectric TEPs shunted externally with electric circuits. All results are validated and verified by means of a three-dimensional (3D) elasticity solver 


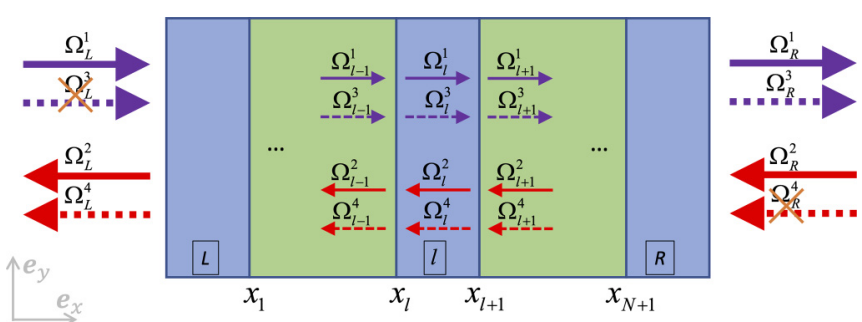

FIG. 1. Scheme showing the multiple layers and the interfaces of a TEP, as well as the incoming and outgoing propagating and evanescent flexural waves. The gray arrows show the $x-y$ plane unit vectors.

using finite-element-method (FEM)-based commercial software COMSOL MULTIPHYSICS [38]. The spectral singularity can be also used for coherent perfect absorber (CPA or antilasing) in elastic plates.

\section{BACKGROUND AND MATHEMATICAL FORMULATION}

In the frequency-domain regime, i.e., assuming an $e^{-i \omega t}$ time dependence and further supposing locally isotropic structures, the equation governing flexural waves in TEPs is given by (see SM [8] for derivation)

$$
\Delta^{2} W-\beta^{4} W=0,
$$

where $W$ is the displacement in the vertical $(z)$ direction, and $\Delta$ represents the Laplacian operator. $\beta$ is the flexural wave number related to angular frequency through the quadratic dispersion $\beta^{2}=\omega \sqrt{\rho \delta / D}$ with $\delta$ the TEP's thickness, $\rho$ its density, and $D$ its flexural rigidity, that is, $E \delta^{3} /\left[12\left(1-v^{2}\right)\right]$ with $E$ and $v$ being its Young's modulus and Poisson's ratio, respectively. The width of these layers (in the $x$ direction as seen in Fig. 1) is assumed to be identical and is set as $20 \mathrm{~cm}$. The thickness of the plate (in the $z$ direction) is set as $2 \mathrm{~cm}$, unless otherwise stated. The other parameters of the plates are taken as follows: densities of 2790 and $7500 \mathrm{~kg} / \mathrm{m}^{3}$, which correspond to a Duraluminium and PZT TEPs, respectively [39]. It should be emphasized here that in all our calculations, we make sure that the flexural wavelength $\lambda$ is always much larger than $\delta$, i.e., $\lambda \gg \delta$. Equation (1) is supplied with four boundary conditions, owing to the order of the PDE, which are continuity of the displacement $W$, its normal derivative $\mathbf{n} \cdot \nabla W=\partial W / \partial n$, the bending moment $M_{n}$, and Kirchhoff's stress $V_{n}$ [8]. Since the structures considered in this work are invariant in the $y$ direction (see Fig. 1) and due to the thin-plate approximation (KL), only the components in the $x$ direction of the above-mentioned parameters contribute to the boundary conditions, i.e., $W, \partial W / \partial x, D \partial^{2} W / \partial x^{2}$, and $D \partial^{3} W / \partial x^{3}$, by omitting the minus sign in the last two expressions.

The solutions of Eq. (1) in the one-dimensional (1D) case (i.e., $d^{4} W / d x^{4}-\beta^{4} W=0$ ) consist of coexisting evanescent (imaginary wave number) and propagating (real wave number) flexural plane waves. Thus, the displacement field in region $l$ (as shown in Fig. 1) can be written as

$$
W_{l}(x)=\Omega_{l}^{1} e^{i \beta_{l} x}+\Omega_{l}^{2} e^{-i \beta_{l} x}+\Omega_{l}^{3} e^{-\beta_{l} x}+\Omega_{l}^{4} e^{\beta_{l} x},
$$

where $\beta_{l}$ denotes the flexural wave number in the layer $l$ and $\Omega_{l}^{i}, i=1, \ldots, 4$ should be determined by the four boundary conditions. The left and right regions are denoted, respectively, by $L$ and $R$ subscripts, so

$$
\begin{aligned}
W_{L / R}(x)= & \Omega_{L / R}^{1} e^{i \beta_{L / R} x}+\Omega_{L / R}^{2} e^{-i \beta_{L / R} x} \\
& +\Omega_{L / R}^{3} e^{-\beta_{L / R} x}+\Omega_{L / R}^{4} e^{\beta_{L / R} x} .
\end{aligned}
$$

The coefficient $\Omega_{L}^{3}$ and $\Omega_{R}^{4}$ are shown only for symmetry reason, but these should be identically zero since they correspond to exponentially diverging terms (as shown in Fig. 1 by the crossed terms). The expression of the boundary conditions at each interface are given in SM [8].

\section{III. $\mathcal{P} \mathcal{T}$-SYMMETRY PROOF-OF-CONCEPT}

\section{A. Piezoelectric realization of gain and loss}

To begin with, a comment about loss and gain conventions in this system should be emphasized: In this work, since a time dependence of $e^{-i \omega t}$ is assumed, one can expect that a positive imaginary part of Young's modulus would account for losses. Yet, by observing the dispersion relation of flexural waves, and since $D$ (and hence $E$ ) appears in the denominator, a positive imaginary part of $E$ would account for gain of the flexural wave number, as

$$
\frac{1}{\operatorname{Re}(E)+i \operatorname{Im}(E)}=\frac{\operatorname{Re}(E)-i \operatorname{Im}(E)}{\operatorname{Re}(E)^{2}+\operatorname{Im}(E)^{2}} .
$$

A shunted piezoelectric TEP [32,33] may lead to an effective isotropic Young's modulus (or flexural rigidity) with a positive (gain) or negative (loss) imaginary part, depending on the use of an inductor and a positive (or negative) resistor (see SM [8]). It is possible to electronically obtain the negative resistance by making use of an active negative-impedance converter (NIC) [40].

Here, we build upon some recent works that have shown the possibility of using shunted external circuits to tailor gain and/or loss in TEPs [35] or beams [36]. For instance, following Ref. [41] it is possible to derive (see SM for more details [8]) the effective equations of the piezoelectric (PZT) elastic layers. In the case of a plate system using the stress tensor that can be written in a vector form $\sigma=\left(\sigma_{x x}, \sigma_{y y}, \sigma_{x y}\right)^{T}$ and the strain tensor $\varepsilon=\left(\varepsilon_{x x}, \varepsilon_{y y}, \gamma_{x y}\right)^{T}$, these equations are

$$
\left(\begin{array}{c}
\varepsilon_{x x} \\
\varepsilon_{y y} \\
\gamma_{x y}
\end{array}\right)=\left(\begin{array}{ccc}
s_{x x}^{\prime} & s_{x y}^{\prime} & 0 \\
s_{x y}^{\prime} & s_{x x}^{\prime} & 0 \\
0 & 0 & 2\left(s_{x x}^{\prime}-s_{x y}^{\prime}\right)
\end{array}\right)\left(\begin{array}{c}
\sigma_{x x} \\
\sigma_{y y} \\
\sigma_{x y}
\end{array}\right),
$$

with

$$
s_{x x}^{\prime}=s_{x x}^{E}-\frac{i \omega d_{x z}^{2} \tilde{Z}}{\delta+i \omega \epsilon_{z z}^{T} \tilde{Z}},
$$

and

$$
s_{x y}^{\prime}=s_{x y}^{E}-\frac{i \omega d_{x z}^{2} \tilde{Z}}{\delta+i \omega \epsilon_{z z}^{T} \tilde{Z}},
$$

with $\tilde{Z}=S Z, S$ is the area of the piezoelectric patch and the impedance $Z=R+i \omega L$, where $R$ and $L$ are the resistance and inductance shunted externally, respectively. Here, we consider (unless otherwise specified) the parameters of PZT are 

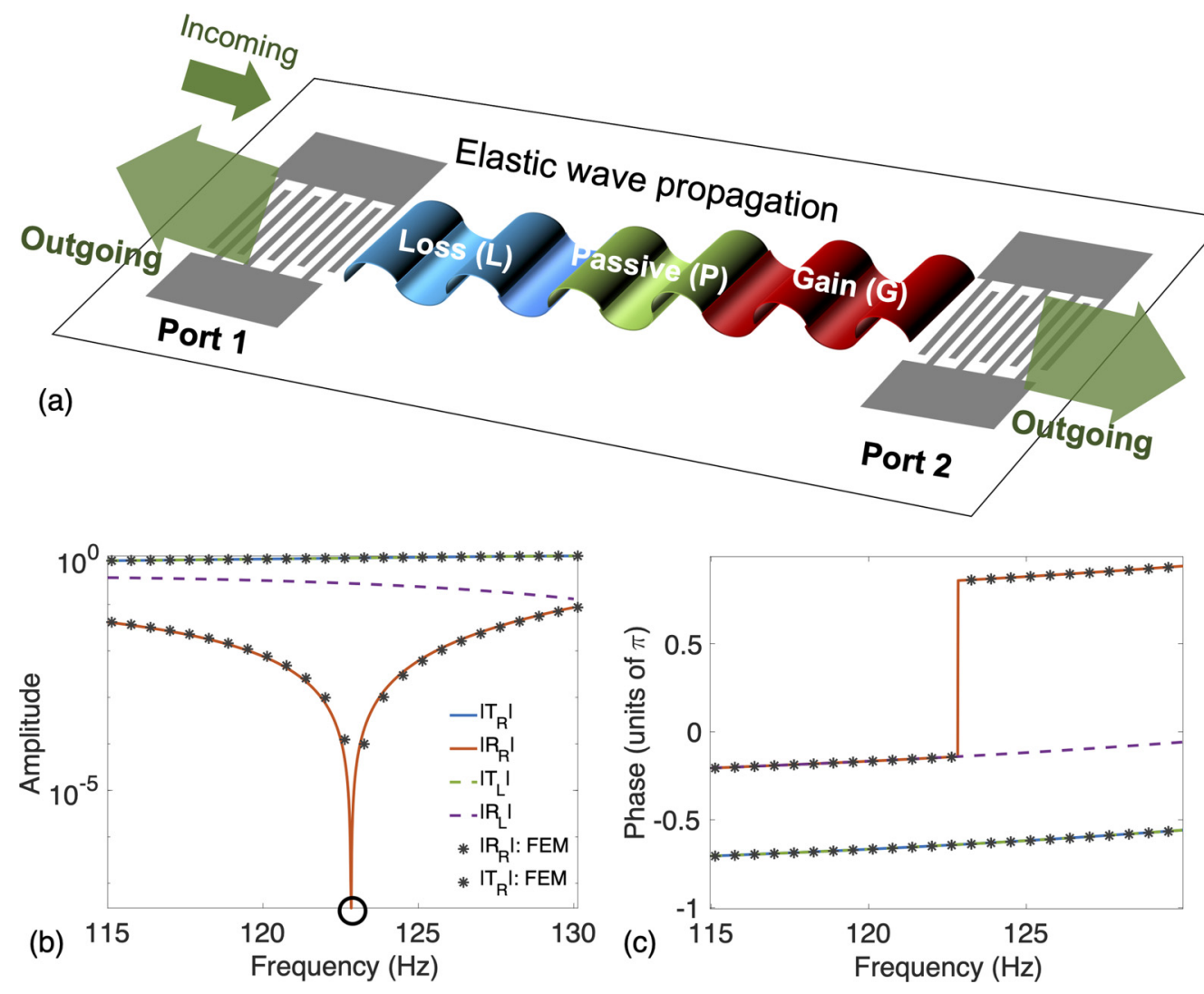

FIG. 2. (a) Proposed device when operating at the lasing point. The size of the arrows is proportional to the strength of the wave. (b) Amplitude of the (left/right) transmittance/reflectance of the structure, in logarithmic scale. (c) Phase of the (left/right) transmittance/reflectance of the same structure, in units of $\pi$. All three layers have the same width of $20 \mathrm{~cm}$. The thickness of the TEPs is equal to $2 \mathrm{~cm}$, its Poisson's ratio is equal to 0.334 , its density to $2790 \mathrm{~kg} / \mathrm{m}^{3}$, and its Young's modulus is equal to $70 \mathrm{GPa}$. In the active/lossy layers, i.e., PZT, the density is $7500 \mathrm{~kg} / \mathrm{m}^{3}$, while Young's modulus and Poisson's ratio are taken from the effective parameters, of Eqs. (6) and (7), and its thickness is $0.5 \mathrm{~cm}$. The dark stars indicate the results obtained by using 3D FEM COMSOL results (see Appendix C).

$d_{x z}=-2.74 \times 10^{-10} \mathrm{C} / \mathrm{N}, s_{x x}^{E}=1.65 \times 10^{-11} \mathrm{~Pa}^{-1}, s_{x y}^{E}=$ $-4.78 \times 10^{-12} \mathrm{~Pa}^{-1}$, and $\epsilon_{z z}^{T}=2000 \epsilon_{0}$ where $\epsilon_{0}=8.854 \times$ $10^{-12} \mathrm{~F} / \mathrm{m}$ is the permittivity of free space $[35,36,41]$. Owing to Eq. (4) and Eq. (6) a positive (negative) value of $R$ corresponds to loss (gain) (see SM [8]). It is indeed further noted that these equations have the same form as the equations for the isotropic nonpiezoelectric thin plate, which means the shunted PZT TEP can be treated as the effective isotropic nonpiezoelectric plate with an equivalent Young's modulus $E^{\mathrm{eff}}=1 / s_{x x}^{\prime}($ see SM [8]).

\section{B. Proof-of-concept demonstration}

The structures we consider [schematized in Fig. 2(a)] consist of three elastic layers denoted as G, L, and P, which stand for gain, loss, and passive, respectively. The possible realization of gain and/or loss in such elastic structures is discussed in the previous section. Using Eq. (B3) of Appendix B we compute the reflection and transmission spectra of this structure when a flexural plane wave is impinging from the left and/or the right. The results are depicted in Figs. 2(b) and 2 (c) in the frequency range $115-130 \mathrm{~Hz}$ (which corresponds to flexural wavelengths in the surrounding medium in the range $121.8-129.5 \mathrm{~cm}$, i.e., $\lambda \gg \delta$ ). Since $\mathcal{P} \mathcal{T}$-symmetric wave systems are reciprocal, the transmittance is the same for wave incident from both directions. However, for this specific $\mathcal{P} \mathcal{T}$ symmetric scenario, the reflectance is drastically different for the right $\left(r_{R}\right)$ and left $\left(r_{L}\right)$ incidences as shown in Fig. 2(b). Here we denote $R=|r|^{2}$ and $T=|t|^{2}$.

For instance, $R_{L}$ is nonzero for essentially all the spectral range considered here, whereas $R_{R}$ undergoes a resonant effect and vanishes around $122.8 \mathrm{~Hz}$. By inspection of the behavior of the phase shown in Fig. 2(c), we can clearly see that the phase of $R_{R}$ is characterized by a jump around the same frequency of $122.8 \mathrm{~Hz}$. This phase jump of $\pi$ confirms the resonant asymmetric reflection from the $\mathcal{P} \mathcal{T}$-symmetric system. The dark stars in Figs. 2(b) and 2(c) give the numerical results obtained by making use of FEM that solves the full elasticity equation in three dimensions (see Appendix C), using COMSOL MULTIPHYSICS [38]. The two models agree very well in the considered frequency range, as the wavelength is around $1 \mathrm{~m}$, i.e., $\lambda \gg \delta$ and hence the KL plate model works well (only a small shift of the frequency can be observed of less than $0.15 \mathrm{~Hz}$ ).

In order to understand this peculiar behavior (asymmetric reflection), we check the scattering matrix of the system. The reflection and transmission are computed from Eq. (B3) and denoted as $r$ and $t$, without the subscripts, since only one type of flexural wave contributes to the far-field scattering. Then, these coefficients can be used to define the scattering matrix 

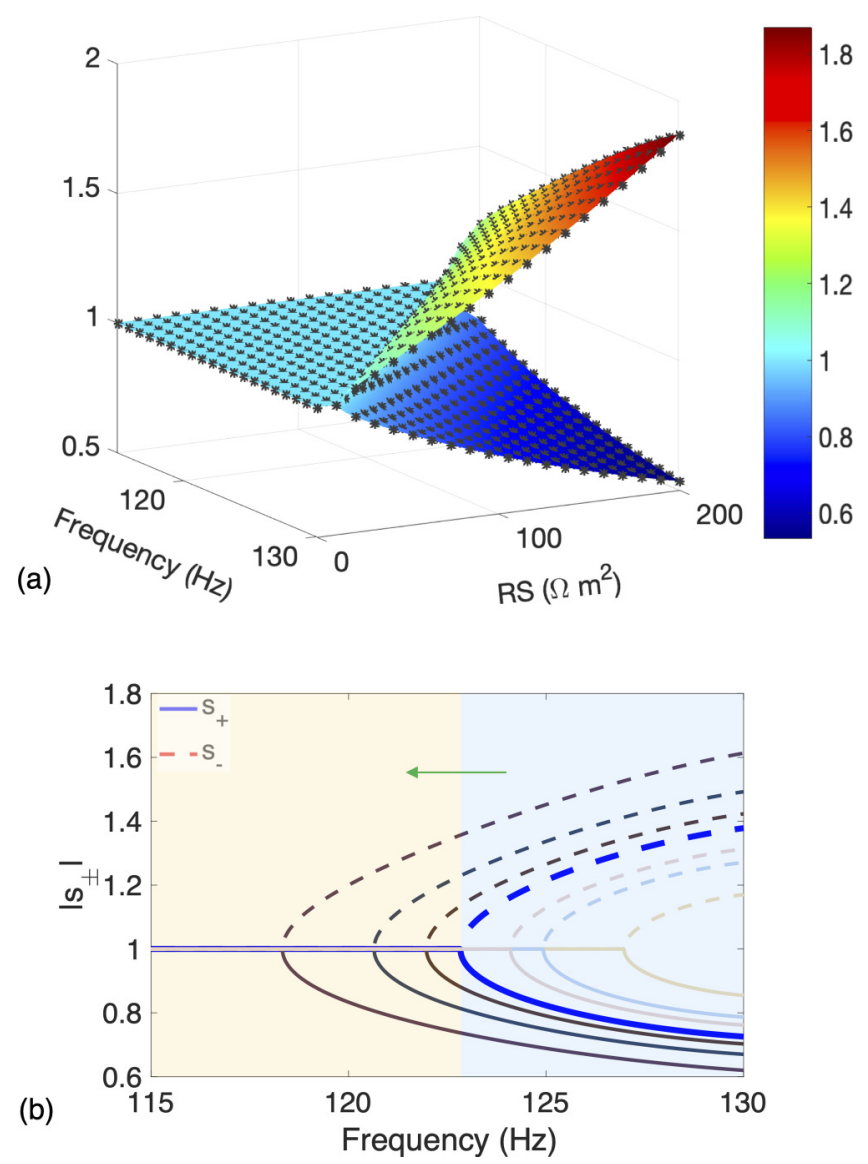

FIG. 3. (a) Contour plot of the amplitude of the eigenvalues versus frequency and $R S$. The dark stars give the results obtained by using FEM full 3D elasticity. (b) Eigenvalues for (increasing with arrow's direction) loss/gain values, i.e., $R S=(50,75,85,100,105$, 110,125 , and 150) $\Omega \mathrm{m}^{2}$. Thick lines correspond to $R S=100 \Omega \mathrm{m}^{2}$. The inductance of the piezoelectric layer is $L S=0.1 \mathrm{Hm}^{2}$. The thickness of the layers is equal to $2 \mathrm{~cm}$, the density and Poisson's ratio of the passive layers are $2790 \mathrm{~kg} / \mathrm{m}^{3}$ and 0.334 , respectively, while the density of the gain/loss layers is $7500 \mathrm{~kg} / \mathrm{m}^{3}$ and their thickness is $0.5 \mathrm{~cm}$.

(in terms of solely the propagating waves) as follows:

$$
\tilde{S}(\beta)=\left(\begin{array}{cc}
t & r_{R} \\
r_{L} & t
\end{array}\right),
$$

where $r_{R}$ and $r_{L}$ denote the reflection coefficient from the right and the left, respectively. The matrix of Eq. (8) obeys the relation $\tilde{S}^{*}(\beta)=\tilde{S}^{-1}(\beta)$, where ${ }^{*}$ denotes the complex conjugate, and hence, it can be deduced that $r_{L} r_{R}^{*}+t t^{*}=1$. The eigenvalues of the scattering matrix $\left(s_{ \pm}\right)$are obtained as function of the $\mathrm{S}$ parameters $\left(t, r_{R}\right.$, and $\left.r_{L}\right)$, i.e., $s_{ \pm}=$ $t \pm \sqrt{r_{L} r_{R}}=t(1 \pm i \sqrt{(1 / T-1)})$

Figures 3(a) and 3(b) depict the absolute value of the two eigenvalues of the scattering matrix (corresponding to propagating waves, i.e., given in Eq. (8) versus the spectral range considered earlier for different values of $R S$. It can be clearly seen from the two-dimensional (2D) plot of Fig. 3(a) that for each value of $R S$ there exists a different frequency, where the spontaneous symmetry breaking takes place (or equivalently, the EP occurs). Moreover, the dark stars show the numerical results obtained again using FEM and demonstrate a good agreement between two models (i.e., using FEM and TMM: transfer matrix method). More specifically, let us consider curves with thicker lines in Fig. 3(b) showing the case of Figs. 2(b) and 2(c). For frequencies higher than $122.8 \mathrm{~Hz}$ (blue highlight), we can observe non-unit-modular eigenvalues. This stems from the fact that $|t|^{2}>1$, and the flexural system is thus in the so-called broken phase [26]. On the contrary, for frequencies lower than $122.8 \mathrm{~Hz}$ (yellow highlight), $s_{ \pm}$have both the unit module and are nondegenerate, implying that the system is in the exact phase. Around this critical frequency, a sudden phase change occurs when the $\mathcal{P} \mathcal{T}$-symmetric structure flips from a broken- $\mathcal{P} \mathcal{T}$ to a $\mathcal{P} \mathcal{T}$ symmetric domain: An EP takes place. For a small value of $R S=50 \Omega \mathrm{m}^{2}$, the EP frequency is around $127 \mathrm{~Hz}$, while for $R S=150 \Omega \mathrm{m}^{2}$, a redshift close to $117 \mathrm{~Hz}$ can be observed. This behavior is confirmed by observing the phase of $r_{L}$, that undergoes an abrupt jump of $\pi$ radians, around the same frequencies [8], validating the possibility of tuning the EP location by varying $\operatorname{Im}(E)$ of gain/loss layers. Such a large tunability of the EP with the amount of (equal) loss (and/or gain) in $E$ is somehow specific to flexural waves, as in acoustics, for example, the location of the EP is less sensitive to the value of $\operatorname{Im}(\rho)$ [42]. More detailed analysis of the peculiarity of flexural $\mathcal{P} \mathcal{T}$-symmetric systems is given in SM [8] and showcases more degrees of freedom to tune and thus control the location and even shape of the EP zone, essentially due to its parabolic dispersion relation and the coupling between propagating and evanescent waves at the interfaces between the gain/loss layers, which differentiates its physics from other waves.

\section{CPAL FOR ELASTICITY}

\section{A. Peculiarity of flexural $\mathcal{P} \mathcal{T}$-symmetry: Interplay of propagating and evanescent waves}

Flexural waves propagating within a piezoelectric TEP were shown to obey the KL equation, i.e., Eq. (1) in the frequency domain. In addition to propagating flexural waves, i.e., $e^{ \pm i \beta x}$, there exist evanescent (inhomogeneous) flexural waves, differentiating the TEP from its acoustic counterpart, in which only propagating waves are considered. In a free propagating domain, only the propagating component survives as shown in Eq. (3) where the evanescent wave is proportional to $e^{ \pm \beta_{L, R} x}$ for negative and positive $x$, respectively. Since these evanescent waves decay exponentially as they travel away from their corresponding interfaces, they do not contribute to the scattering coefficients, which are measured in the far field. Yet, in order to fully characterize the scattering of flexural waves, we have to take into account the contribution of all kinds of waves at the inner interfaces (shown in Fig. 1). What is more intriguing is that evanescent waves establish propagating components, in the presence of gain and loss. This behavior is unlike the case of elastic plates without loss and/or gain, where the evanescent waves are confined to interfaces.

\section{B. Analysis of the CPAL effect}

Inspired by this behavior of EP for flexural waves, we consider the possibility of CPAL effect. It is well known that 

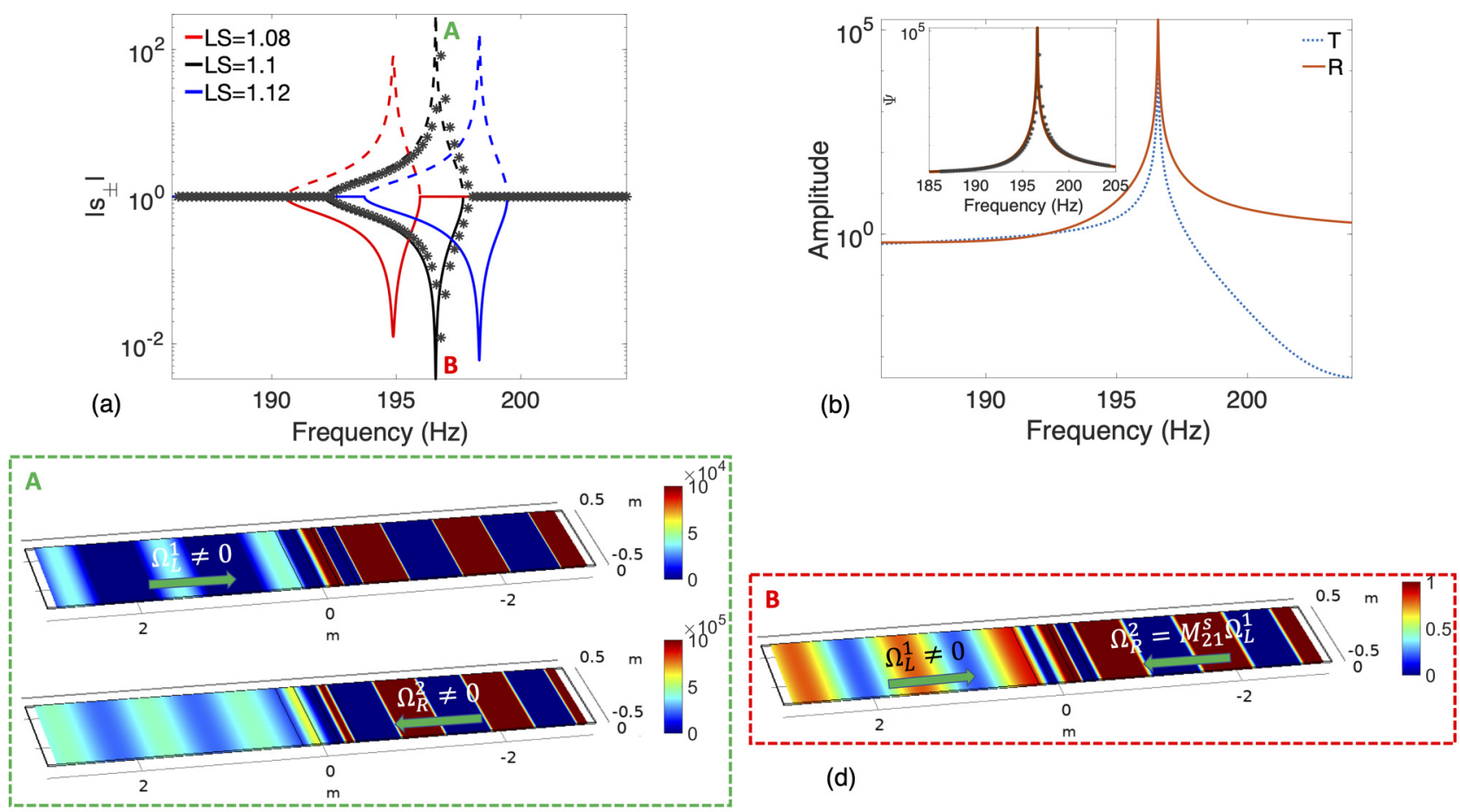

(d)

(c)

FIG. 4. (a) Amplitude of the eigenvalues in the frequency domain where CPAL takes place for $L S=1.08,1.1$, and $1.12 \mathrm{Hm}^{2}$ (red, black, and blue, respectively). For the higher frequency regime, see SM [8]. Points A and B indicate lasing and perfect absorption operation, respectively. (b) Transmittance and reflectance from the CPAL structure. The inset plots the output coefficient $\Psi$ given in Eq. (9). The dark star curves are FEM-based results (obtained using the 3D full elasticity module of COMSOL [38]). (c) Numerically computed (3D FEM) snapshots of flexural energy $|W|^{2}$ for the FLASER in (a) at operating frequency $196.6 \mathrm{~Hz}$ (Point A) when the incident wave impinges from left (top) and right (bottom). (d) Same as in (c) but for the CPA mode (Point B) at the same frequency with $\Omega_{R}^{2}=M_{21}^{s} \Omega_{L}^{1}$. The colorbars are normalized by the amplitude of the incident waves.

in optics, $\mathcal{P} \mathcal{T}$-symmetric systems can operate as coherent perfect absorbers by totally absorbing the incoming energy (from impinging waves) and as lasers by emitting coherently outgoing waves [43-45]. These two phenomena can be characterized by the overall output coefficient

$$
\Psi=\frac{\left|\Omega_{L}^{2}\right|^{2}+\left|\Omega_{R}^{1}\right|^{2}}{\left|\Omega_{L}^{1}\right|^{2}+\left|\Omega_{R}^{2}\right|^{2}},
$$

which accounts for the ratio of total outgoing intensity to that of the incoming waves. Although absorbing and even perfectly absorbing structures using different mechanisms have been widely studied in acoustics [46-48] and elasticity [49,50], the effects of lasing and CPAL effect for elastic plates have not been investigated to the best of our knowledge. For instance, to obtain efficient lasing, we maintain a similar structure as before (see Fig. 3) and apply higher inductance, as well as blueshifting frequencies. The result is plotted in Fig. 4(a), which depicts the eigenvalues $\left|s_{ \pm}\right|$for frequencies $185-205 \mathrm{~Hz}$. The plot is given for three values of $L S(1.08$, 1.1 , and 1.12$) \mathrm{Hm}^{2}$ and $\left|s_{-}\right|$can reach the lasing regime, with $\left|s_{-}\right| \approx 10^{3}$, at frequency around $196.6 \mathrm{~Hz}$ (denoted A). The complementary eigenvalue $\left|s_{+}\right| \approx 0$ is reminiscent of the CPA regime as in fact we have $s_{+}=1 / s_{-}^{*}$. Thus, at the same frequency, we have the lasing regime $\left(s_{-}\right)$where the outgoing energy is hugely amplified and the CPA regime $\left(s_{+}\right)$where the outgoing energy is canceled (i.e., the all impinging signal is absorbed by the flexural system).

Figure 4(b) shows the transmittance $T$ and reflectance $R$ spectra for a unit-amplitude flexural wave incidence. Both $T$ and $R$ reach extremely high values at the frequency of Point $\mathrm{A}$, i.e., $196.6 \mathrm{~Hz}$. The coefficient $\Psi$ shown in the inset of Fig. 4(b) gives a better picture of the lasing efficiency of the device. At the same frequency the spike reaching $10^{5}$ manifests the lasing effect. All these results are validated by the FEM model and show good agreement as can be seen by the dark star curves. To further demonstrate this effect, in Fig. 4(c) we plot the flexural energy computed by COMSOL MULTIPHYSICS [38] in the vicinity of the CPAL device where a normally incident flexural wave of unit amplitude is impinging from the left (top panel) and the right (bottom panel). In both cases, the scattered waves (both transmitted and reflected) are significantly amplified (in the range of $10^{5}$ ). However, for one scenario (left incidence) the transmittance is higher than the reflectance. For the other scenario (right incidence) the reflectance dominates as can be seen from the plots.

These results demonstrate the potential of using a simple and compact (60 $\mathrm{cm}$ total width and $2 \mathrm{~cm}$ thickness) flexural system to achieve the equivalent of a flexural laser that we might coin a FLASER. Consider a flexural wave with very small vertical displacement, of amplitude $|W| \approx 10 \mu$ m (i.e., $|W| \ll \delta$ ) incident on the CPAL. Although this signal is very 
small, it will be amplified by the CPAL flexural device, to the point that the output displacement will be in the range of $1 \mathrm{~cm}$, i.e., $|W| \approx \delta$. Now to relate this effect to the transfer matrix (see Appendix B) it is straightforward to see that lasing may occur, when we have finite (propagating) outgoing signals $\Omega_{L}^{2}$ and $\Omega_{R}^{1}$ for very small incoming signals. This may occur for $M_{22}^{s}=0$ (and the outgoing waves are related through $M_{12}^{s}=\Omega_{R}^{1} / \Omega_{L}^{2}$ ) without considering the evanescent fields, as can be concluded from Eqs. (B3) and (B4) in Appendix B. Yet, this is generally not possible, as the evanescent fields $\Omega_{L}^{4}$ and $\Omega_{R}^{3}$ cannot be assumed to be zero at the boundary of the system. Hence, one must include the intermediate evanescent waves in deriving the scattering properties of the system. The deduced reflection coefficients, for a left incidence (without loss of generality) can be obtained [8] from the terms of the $S$ matrix, i.e., those of the first column that give the reflection/transmission coefficients of both propagating and evanescent waves, and that can be explicitly given by

$$
r_{1}=S_{11}=\frac{\left|\begin{array}{ll}
-M_{21}^{s} & M_{24}^{s} \\
-M_{41}^{s} & M_{44}^{s}
\end{array}\right|}{\Delta M^{s}} ; \quad r_{2}=S_{21}=\frac{\left|\begin{array}{ll}
M_{22}^{s} & -M_{21}^{s} \\
M_{42}^{s} & -M_{41}^{s}
\end{array}\right|}{\Delta M^{s}},
$$

where $|\cdot|$ is the determinant function and $\Delta M^{s}=M_{22}^{s} M_{44}^{s}-$ $M_{42}^{s} M_{24}^{s}$. This system has to satisfy the general condition that for a unit-amplitude incident signal $\left(\Omega_{L}^{1}=1\right)$ the outgoing signals diverge. Equivalently, if the incidence is taken near zero and if we require finite scattering signals, as occurs in lasing, we must ensure that the denominator of the expressions in Eq. (10) is zero, which yields $\Delta M^{s}=0$, which is markedly different from the simple condition $M_{22}^{s}=0$ for acoustic or optical systems, for example [see Fig. 7(b) in SM [8] for the comparison of both conditions]. For the transmitted signals, it is easy to obtain their expressions (see Appendix B), i.e., $t_{1}=S_{31}=M_{11}^{s}+M_{12}^{s} S_{11}+M_{14}^{s} S_{21}$.

\section{DISCUSSION}

The complexity of flexural CPAL, discussed in the previous section, stems thus from the interplay between propagating and evanescent waves that cannot be ignored for flexural systems, as clearly demonstrated by the lasing equation that intertwines amplitudes of both kinds of waves. The variation of the parameter $\Psi \propto 1 /\left(\Delta M^{s}\right)^{2}$ responsible for huge outgoing amplitudes (i.e., FLASER) is depicted in Fig. 5 versus the frequency of the flexural wave and $L S$. The dark red regions correspond to the FLASER regime (i.e., $\Delta M^{s} \approx 0$ ). We can clearly see that if FLASER is defined when the logarithmic amplitude of the lasing parameter is above $30 \mathrm{~dB}$, a narrow lasing region (interval) in terms of $L S$ can be defined for each frequency, below (above) which no lasing can occur. From this landscape of $L S$ we find that this behavior is hyperbolic (dark red region). The magnified view given in the inset of Fig. 5 further shows that the FLASER effect takes place indeed at discrete locations shaped as a vertical rhombus. This means that the FLASER effect is extremely sensitive to frequency, and slightly less sensitive to the external parameter $L S$, giving some freedom to experimental design. A similar reasoning can be made for the CPA ef-

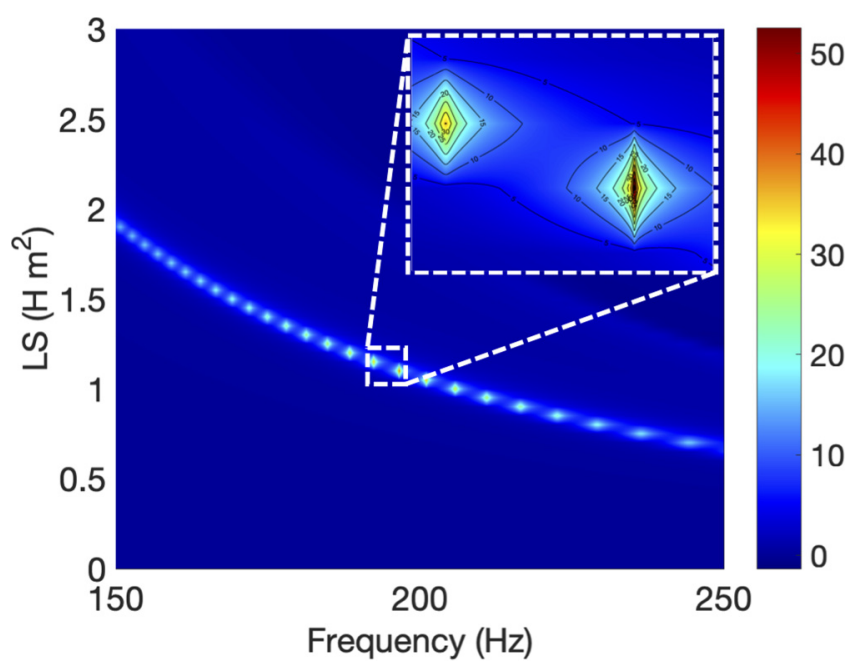

FIG. 5. Contour plot of $\Psi$ versus frequency and $L S$ in logarithmic scale, i.e., $10 \log _{10}(\Psi)$. Dark red regions correspond to the lasing regime $\left(\Delta M^{s} \approx 0\right)$. The inset shows a magnified view marked by the white dashed square.

fect. In fact, for perfect absorption to occur, we must cancel the outgoing waves for finite incoming waves. The same analysis as before shows that for the CPA to take place, we must ensure that $M_{11}^{s} \Omega_{L}^{1}+M_{13}^{s} \Omega_{L}^{3}=0$ and $M_{21}^{s} \Omega_{L}^{1}+$ $M_{23}^{s} \Omega_{L}^{3}=\Omega_{R}^{2}$. Yet, in the CPA case, $\Omega_{L}^{3}=0$ as it corresponds to an exponentially growing field when $x \rightarrow-\infty$. Thus for the CPA operation, evanescent waves are not directly present in the condition on the amplitudes. However, their indirect effect on the $M$ matrix is still present. Therefore, we need to launch two waves incoming from opposite directions, and by changing their amplitude and phase ratio, we will be able to selectively excite the lasing or CPA mode, as can be seen in Fig. 4(d). If their complex ratio $M_{21}^{s}=\Omega_{R}^{2} / \Omega_{L}^{1}$, we will achieve the CPA operation mode. In the rest of the cases, i.e., $M_{21}^{s} \neq \Omega_{R}^{2} / \Omega_{L}^{1}$ we will have the FLASER mode. This is, however, a very narrow-band effect for both CPA and lasing, as seen in Figs. 4(a) and 5.

\section{CONCLUSIONS}

To sum up, an effect reminiscent of lasing is discovered in this paper by making use of $\mathcal{P} \mathcal{T}$-symmetry in a specific frequency and gain/loss regimes. This mechanism takes roots in the CPAL effect, first proposed for optics [43]. This CPAL device can be used as an ultrasensitive flexural sensor to detect submicrometer displacements or as a perfect absorber of flexural energy. However, in stark contrast to Maxwell's equations, the flexural wave equation assumes displacements that are small in comparison with the thickness of the plate, so our device could be used as a source, since by applying very small displacements, these will be amplified and coherently transmitted to the surroundings. On the detection side, if some strong signal/noise impinges on the device, it can be hugely amplified and can lead to its dislocation. One way to avoid this unwanted effect is to use a filter that cancels out incoming signals above a certain threshold. Our work can thus pave the way to several interesting applications in surface science and civil engineering, e.g., in precision-displacement sensing, vibration control of mechanical systems, and seismic energy harvesting. 


\section{ACKNOWLEDGMENTS}

The research reported in this manuscript was supported by KAUST Office of Sponsored Research (OSR) under Grant No. OSR-2016-CRG5-2950 and KAUST Baseline Research Fund BAS/1/1626-01-01. The authors would like also to acknowledge comments from anonymous reviewers that helped to substantially improve the quality of the paper.

\section{APPENDIX A: TRANSFER MATRIX FORMALISM}

When we need to compute the transfer matrix corresponding to the layer $l$ shown in Fig. 1, we only have to relate the column vector $\mathbf{W}_{l}\left(x_{l}\right)=$ $\left(W_{l}, d W_{l} / d x, D_{l} d^{2} W_{l} / d x^{2}, D_{l} d^{3} W_{l} / d x^{3}\right)^{T}$ to $\mathbf{W}_{l}\left(x_{l+1}\right)$, as $\mathbf{W}_{l+1}\left(x_{l+1}\right)=\mathbf{W}_{l}\left(x_{l+1}\right)$, through the continuity condition at the interface $x_{l+1}$. This consists thus in finding the propagator of these flexural waves. For this, let us give the set of matrices, defined at the coordinates $x_{l}$ and $x_{l+1}$,

$$
M_{l}^{l}=\left(\begin{array}{cccc}
\left(i \beta_{l}\right)^{0} e^{i \beta_{l} x_{l}} & \left(-i \beta_{l}\right)^{0} e^{-i \beta_{l} x_{l}} & \left(-\beta_{l}\right)^{0} e^{-\beta_{l} x_{l}} & \left(\beta_{l}\right)^{0} e^{\beta_{l} x_{l}} \\
\left(i \beta_{l}\right)^{1} e^{i \beta_{l} x_{l}} & \left(-i \beta_{l}\right)^{1} e^{-i \beta_{l} x_{l}} & \left(-\beta_{l}\right)^{1} e^{-\beta_{l} x_{l}} & \left(\beta_{l}\right)^{1} e^{\beta_{l} x_{l}} \\
D_{l}\left(i \beta_{l}\right)^{2} e^{i \beta_{l} x_{l}} & D_{l}\left(-i \beta_{l}\right)^{2} e^{-i \beta_{l} x_{l}} & D_{l}\left(-\beta_{l}\right)^{2} e^{-\beta_{l} x_{l}} & D_{l}\left(\beta_{l}\right)^{2} e^{\beta_{l} x_{l}} \\
D_{l}\left(i \beta_{l}\right)^{3} e^{i \beta_{l} x_{l}} & D_{l}\left(-i \beta_{l}\right)^{3} e^{-i \beta_{l} x_{l}} & D_{l}\left(-\beta_{l}\right)^{3} e^{-\beta_{l} x_{l}} & D_{l}\left(\beta_{l}\right)^{3} e^{\beta_{l} x_{l}}
\end{array}\right)
$$

and

$$
M_{l}^{l+1}=\left(\begin{array}{cccc}
\left(i \beta_{l}\right)^{0} e^{i \beta_{l} x_{l+1}} & \left(-i \beta_{l}\right)^{0} e^{-i \beta_{l} x_{l+1}} & \left(-\beta_{l}\right)^{0} e^{-\beta_{l} x_{l+1}} & \left(\beta_{l}\right)^{0} e^{\beta_{l} x_{l+1}} \\
\left(i \beta_{l}\right)^{1} e^{i \beta_{l} x_{l+1}} & \left(-i \beta_{l}\right)^{1} e^{-i \beta_{l} x_{l+1}} & \left(-\beta_{l}\right)^{1} e^{-\beta_{l} x_{l+1}} & \left(\beta_{l}\right)^{1} e^{\beta_{l} x_{l+1}} \\
D_{l}\left(i \beta_{l}\right)^{2} e^{i \beta_{l} x_{l+1}} & D_{l}\left(-i \beta_{l}\right)^{2} e^{-i \beta_{l} x_{l+1}} & D_{l}\left(-\beta_{l}\right)^{2} e^{-\beta_{l} x_{l+1}} & D_{l}\left(\beta_{l}\right)^{2} e^{\beta_{l} x_{l+1}} \\
D_{l}\left(i \beta_{l}\right)^{3} e^{i \beta_{l} x_{l+1}} & D_{l}\left(-i \beta_{l}\right)^{3} e^{-i \beta_{l} x_{l+1}} & D_{l}\left(-\beta_{l}\right)^{3} e^{-\beta_{l} x_{l+1}} & D_{l}\left(\beta_{l}\right)^{3} e^{\beta_{l} x_{l+1}}
\end{array}\right) .
$$

Thus we have the following relation:

$$
\mathbf{W}_{l}\left(x_{l+1}\right)=M_{l}^{l+1}\left(\begin{array}{c}
\Omega_{l}^{1} \\
\Omega_{l}^{2} \\
\Omega_{l}^{3} \\
\Omega_{l}^{4}
\end{array}\right)
$$

and

$$
\mathbf{W}_{l}\left(x_{l}\right)=M_{l}^{l}\left(\begin{array}{c}
\Omega_{l}^{1} \\
\Omega_{l}^{2} \\
\Omega_{l}^{3} \\
\Omega_{l}^{4}
\end{array}\right)
$$

Hence, it is straightforward to derive

$$
\mathbf{W}_{l}\left(x_{l+1}\right)=M_{l}^{l+1}\left(M_{l}^{l}\right)^{-1} \mathbf{W}_{l}\left(x_{l}\right),
$$

where the associated transfer matrix of layer $l$ is $\Pi_{l}=$ $M_{l}^{l+1}\left(M_{l}^{l}\right)^{-1}$. If we have $N$ layers, we must first take the product of all these matrices, i.e., $\Pi_{s}=\Pi_{N} \cdot \Pi_{N-1} \cdots \Pi_{l} \cdots \Pi_{1}$. If we define also the incoming and outgoing matrices in the same way, we can then compute the overall transfer matrix of the multilayered structure, i.e.,

$$
M^{s}=\left(M_{\mathrm{out}}^{N+1}\right)^{-1} \Pi_{s} M_{\mathrm{in}}^{1} .
$$

The matrix $M^{s}$ is $4 \times 4$ and obviously different from transfer matrices in electrodynamics and acoustics that are $2 \times 2$. The main difference comes from the existence of evanescent waves, localized only at the interfaces between layers.

\section{APPENDIX B: SCATTERING MATRIX FORMALISM}

We wish to characterize the kind of structures given in Fig. 1, when a flexural plane wave impinges on it. To do so, we need to relate the outgoing waves to the incoming ones, or in a sense the coefficients of the displacement fields in these two regions. In fact, the transfer matrix ensures that

$$
M^{s}\left(\begin{array}{c}
\Omega_{L}^{1} \\
\Omega_{L}^{2} \\
\Omega_{L}^{3} \\
\Omega_{L}^{4}
\end{array}\right)=\left(\begin{array}{c}
\Omega_{R}^{1} \\
\Omega_{R}^{2} \\
\Omega_{R}^{3} \\
\Omega_{R}^{4}
\end{array}\right)
$$

By writing down this linear system and rearranging the terms that correspond to incoming and outgoing flexural waves (both propagating and evanescent), we can rewrite it in the following way, by remarking that $\Omega_{L}^{1}, \Omega_{L}^{3}$, $\Omega_{R}^{2}$, and $\Omega_{R}^{4}$ are the incoming coefficients and $\Omega_{L}^{2}, \Omega_{L}^{4}, \Omega_{R}^{1}$, and $\Omega_{R}^{3}$ are the outgoing coefficients,

$$
\left(\begin{array}{cccc}
M_{11}^{s} & M_{13}^{s} & 0 & 0 \\
M_{21}^{s} & M_{23}^{s} & -1 & 0 \\
M_{31}^{s} & M_{33}^{s} & 0 & 0 \\
M_{41}^{s} & M_{43}^{s} & 0 & -1
\end{array}\right)\left(\begin{array}{l}
\Omega_{L}^{1} \\
\Omega_{L}^{3} \\
\Omega_{R}^{2} \\
\Omega_{R}^{4}
\end{array}\right)=\left(\begin{array}{cccc}
-M_{12}^{s} & -M_{14}^{s} & 1 & 0 \\
-M_{22}^{s} & -M_{24}^{s} & 0 & 0 \\
-M_{32}^{s} & -M_{34}^{s} & 0 & 1 \\
-M_{42}^{s} & -M_{44}^{s} & 0 & 0
\end{array}\right)\left(\begin{array}{l}
\Omega_{L}^{2} \\
\Omega_{L}^{4} \\
\Omega_{R}^{1} \\
\Omega_{R}^{3}
\end{array}\right) .
$$


Without loss of generality, let us further assume that we excite this system from the left side by propagating waves and that we are interested in characterizing the reflection and transmission of each kind of flexural wave. This signifies that we excite by a unitary incident plane wave or $\Omega_{L}^{1}=1$ and $\Omega_{L}^{3}=\Omega_{R}^{2}=\Omega_{R}^{4}=0$. Also by this definition, we have the reflection (transmission) coefficients $r_{1}$ and $r_{2}\left(t_{1}\right.$ and $\left.t_{2}\right)$ of the propagating and evanescent waves, respectively. This linear system can be written as

$$
\left(\begin{array}{cccc}
-M_{12}^{s} & -M_{14}^{s} & 1 & 0 \\
-M_{22}^{s} & -M_{24}^{s} & 0 & 0 \\
-M_{32}^{s} & -M_{34}^{s} & 0 & 1 \\
-M_{42}^{s} & -M_{44}^{s} & 0 & 0
\end{array}\right)^{-1}\left(\begin{array}{cccc}
M_{11}^{s} & M_{13}^{s} & 0 & 0 \\
M_{21}^{s} & M_{23}^{s} & -1 & 0 \\
M_{31}^{s} & M_{33}^{s} & 0 & 0 \\
M_{41}^{s} & M_{43}^{s} & 0 & -1
\end{array}\right)\left(\begin{array}{l}
1 \\
0 \\
0 \\
0
\end{array}\right)=\left(\begin{array}{l}
r_{1} \\
r_{2} \\
t_{1} \\
t_{2}
\end{array}\right),
$$

with the scattering matrix expressed as

$$
S=\left(\begin{array}{llll}
-M_{12}^{s} & -M_{14}^{s} & 1 & 0 \\
-M_{22}^{s} & -M_{24}^{s} & 0 & 0 \\
-M_{32}^{s} & -M_{34}^{s} & 0 & 1 \\
-M_{42}^{s} & -M_{44}^{s} & 0 & 0
\end{array}\right)^{-1}\left(\begin{array}{llll}
M_{11}^{s} & M_{13}^{s} & 0 & 0 \\
M_{21}^{s} & M_{23}^{s} & -1 & 0 \\
M_{31}^{s} & M_{33}^{s} & 0 & 0 \\
M_{41}^{s} & M_{43}^{s} & 0 & -1
\end{array}\right)
$$

The important terms of the $S$ matrix, i.e., those of the first column that give the reflection/transmission coefficients of both propagating and evanescent waves, can be explicitly given by

$$
S_{11}=r_{1}=\frac{\left|\begin{array}{ll}
-M_{21}^{s} & M_{24}^{s} \\
-M_{41}^{s} & M_{44}^{s}
\end{array}\right|}{\left|\begin{array}{ll}
M_{22}^{s} & M_{24}^{s} \\
M_{42}^{s} & M_{44}^{s}
\end{array}\right|} \text { and } S_{21}=r_{2}=\frac{\left|\begin{array}{ll}
M_{22}^{s} & -M_{21}^{s} \\
M_{42}^{s} & -M_{41}^{s}
\end{array}\right|}{\left|\begin{array}{ll}
M_{22}^{s} & M_{24}^{s} \\
M_{42}^{s} & M_{44}^{s}
\end{array}\right|}
$$

for the reflected part, where $|A|$ denotes the determinant of a square matrix $A$, and by

$$
\begin{aligned}
& S_{31}=t_{1}=M_{11}^{s}+M_{12}^{s} S_{11}+M_{14}^{s} S_{21} \quad \text { and } \\
& S_{41}=t_{2}=M_{31}^{s}+M_{32}^{s} S_{11}+M_{34}^{s} S_{21},
\end{aligned}
$$

for the transmitted part.

\section{APPENDIX C: 3D FEM COMSOL MODELING}

The time-harmonic Navier equations, which govern the propagation of elastodynamic waves in the plate, are written as

$$
\nabla \cdot[\mathbb{C}: \nabla \mathbf{u}(\mathbf{x})]+\rho \omega^{2} \mathbf{u}(\mathbf{x})=\mathbf{0},
$$

where we have excluded the source term required to generate a plane flexural wave. The unknown in this tensorial equation is the displacement field

$$
\mathbf{u}(\mathbf{x})=\left[u^{(1)}(\mathbf{x}), u^{(2)}(\mathbf{x}), u^{(3)}(\mathbf{x})\right]^{T},
$$

with $\mathbf{x}=\left(x_{1}, x_{2}, x_{3}\right)$ and $\mathbb{C}$ is the rank-4 (symmetric) elasticity tensor with entries $C_{i j k l}=\lambda \delta_{i j} \delta_{k l}+\mu\left(\delta_{i k} \delta_{j l}+\delta_{i l} \delta_{j k}\right)$, $i, j, k, l=1,2,3$, i.e., isotropic, $\lambda$ and $\mu$ being the Lamé parameters, $\rho$ the mass density, and $\omega$ the angular frequency of the wave.
We set stress-free boundary conditions at the top and bottom boundaries of the plate:

$$
(\mathbb{C}: \nabla \mathbf{u}) \cdot \mathbf{n}=(\mathbb{C}: \epsilon(\mathbf{u})) \cdot \mathbf{n}=\mathbf{0},
$$

where $\epsilon(\mathbf{u})$ is the rank-2 strain tensor with entries $\varepsilon_{i j}=$ $1 / 2\left(\partial u_{i} / \partial x_{j}+\partial u_{j} / \partial x_{i}\right)$ and $\mathbf{n}$ is the outward pointing normal to the boundaries. We solve the exact 3D form of Eq. (C1) using the commercial finite element software COMSOL MULTIPHYSICS [38].

In order to excite the structure of Fig. 2(a), we make use of the port boundary condition recently added by COMSOL to its solid mechanics module [38]. We define a displacement in the $z$-direction incident normally towards the layered CPAL structure. On the side boundary we set Floquet-Bloch boundary conditions to ensure continuity of the elastic displacement. The mesh is taken equal to $\lambda_{\min } / 10$, where $\lambda_{\min }$ is the smallest flexural wavelength. Last but not least, before performing the frequency-domain study, a boundary mode analysis is performed to calculate accurately the propagation constant. It should be emphasized that one has to take great care with this modeling as only a slight deviation can make the receiving port totally reflective.

In order to validate our modeling, we compare the results of an analytical situation where a single elastic layer is present, first without loss or gain, and then with loss and gain (see SM [8]).
[1] J. B. Pendry, A. J. Holden, D. J. Robbins, and W. Stewart, Magnetism from conductors and enhanced nonlinear phenomena, IEEE Trans. Microwave Theory Tech. 47, 2075 (1999).

[2] J. B. Pendry, Negative Refraction Makes a Perfect Lens, Phys. Rev. Lett. 85, 3966 (2000).
[3] A. Alù and N. Engheta, Achieving transparency with plasmonic and metamaterial coatings, Phys. Rev. E 72, 016623 (2005).

[4] P.-Y. Chen, J. Soric, and A. Alu, Invisibility and cloaking based on scattering cancellation, Adv. Mater. 24, OP281 (2012). 
[5] Y. Wu, Y. Lai, and Z.-Q. Zhang, Elastic Metamaterials with Simultaneously Negative Effective Shear Modulus and Mass Density, Phys. Rev. Lett. 107, 105506 (2011).

[6] S. P. Timoshenko and S. Woinowsky-Krieger, Theory of Plates and Shells (McGraw-Hill, New York, 1959).

[7] A. Norris and C. Vemula, Scattering of flexural waves on thin plates, J. Sound Vib. 181, 115 (1995).

[8] See Supplemental Material at http://link.aps.org/supplemental/ 10.1103/PhysRevB.103.134101 for the derivation of the flexural wave equation modeling, detailed boundary conditions, equations and derivation of the transfer and scattering matrix formalism for flexural waves, as well as some results on the tunability of the EPs in the framework of flexural waves.

[9] M. Farhat, S. Guenneau, and S. Enoch, Ultrabroadband Elastic Cloaking in Thin Plates, Phys. Rev. Lett. 103, 024301 (2009).

[10] M. Farhat, P.-Y. Chen, H. Bağc1, S. Enoch, S. Guenneau, and A. Alu, Platonic scattering cancellation for bending waves in a thin plate, Sci. Rep. 4, 4644 (2014).

[11] J. Zhu, Y. Liu, Z. Liang, T. Chen, and J. Li, Elastic Waves in Curved Space: Mimicking a Wormhole, Phys. Rev. Lett. 121, 234301 (2018).

[12] M. Dubois, M. Farhat, E. Bossy, S. Enoch, S. Guenneau, and P. Sebbah, Flat lens for pulse focusing of elastic waves in thin plates, Appl. Phys. Lett. 103, 071915 (2013).

[13] M. Farhat, P.-Y. Chen, S. Guenneau, K. N. Salama, and H. Bağc1, Localized surface plate modes via flexural Mie resonances, Phys. Rev. B 95, 174201 (2017).

[14] A. Movchan, N. Movchan, and R. McPhedran, Bloch-Floquet bending waves in perforated thin plates, Proc. R. Soc. A 463, 2505 (2007).

[15] R. McPhedran, A. Movchan, N. Movchan, M. Brun, and M. Smith, Parabolic trapped modes and steered Dirac cones in platonic crystals, Proc. R. Soc. A 471, 20140746 (2015).

[16] C. M. Bender and S. Boettcher, Real Spectra in Non-Hermitian Hamiltonians Having PT Symmetry, Phys. Rev. Lett. 80, 5243 (1998).

[17] A. Mostafazadeh, Pseudo-Hermiticity versus PT-symmetry. II. A complete characterization of non-Hermitian Hamiltonians with a real spectrum, J. Math. Phys. 43, 2814 (2002).

[18] K. G. Makris, R. El-Ganainy, D. N. Christodoulides, and Z. H. Musslimani, Beam Dynamics in PT Symmetric Optical Lattices, Phys. Rev. Lett. 100, 103904 (2008).

[19] C. E. Rüter, K. G. Makris, R. El-Ganainy, D. N. Christodoulides, M. Segev, and D. Kip, Observation of parity-time symmetry in optics, Nat. Phys. 6, 192 (2010).

[20] L. Feng, R. El-Ganainy, and L. Ge, Non-Hermitian photonics based on parity-time symmetry, Nat. Photonics 11, 752 (2017).

[21] M. Sakhdari, M. Farhat, and P.-Y. Chen, PT-symmetric metasurfaces: Wave manipulation and sensing using singular points, New J. Phys. 19, 065002 (2017).

[22] P.-Y. Chen and J. Jung, PT Symmetry and Singularity-Enhanced Sensing Based on Photoexcited Graphene Metasurfaces, Phys. Rev. Appl. 5, 064018 (2016).

[23] L. Chang, X. Jiang, S. Hua, C. Yang, J. Wen, L. Jiang, G. Li, G. Wang, and M. Xiao, Parity-time symmetry and variable optical isolation in active-passive-coupled microresonators, Nat. Photonics 8, 524 (2014).

[24] B. Peng, Ş. K. Özdemir, F. Lei, F. Monifi, M. Gianfreda, G. L. Long, S. Fan, F. Nori, C. M. Bender, and L. Yang, Parity-time- symmetric whispering-gallery microcavities, Nat. Phys. 10, 394 (2014).

[25] L. Feng, Z. J. Wong, R.-M. Ma, Y. Wang, and X. Zhang, Singlemode laser by parity-time symmetry breaking, Science 346, 972 (2014).

[26] X. Zhu, H. Ramezani, C. Shi, J. Zhu, and X. Zhang, PTSymmetric Acoustics, Phys. Rev. X 4, 031042 (2014).

[27] J. Christensen, M. Willatzen, V. R. Velasco, and M.-H. Lu, Parity-Time Synthetic Phononic Media, Phys. Rev. Lett. 116, 207601 (2016).

[28] H.-x. Li, M. Rosendo-López, Y.-f. Zhu, X.-d. Fan, D. Torrent, B. Liang, J.-c. Cheng, J. Christensen et al., Ultrathin acoustic parity-time symmetric metasurface cloak, Research 2019, 8345683 (2019).

[29] R. Fleury, D. Sounas, and A. Alu, An invisible acoustic sensor based on parity-time symmetry, Nat. Commun. 6, 5905 (2015).

[30] J. Zhang, B. Peng, Ş. K. Özdemir, K. Pichler, D. O. Krimer, G. Zhao, F. Nori, Y.-x. Liu, S. Rotter, and L. Yang, A phonon laser operating at an exceptional point, Nat. Photonics 12, 479 (2018).

[31] L. Quan, D. L. Sounas, and A. Alù, Nonreciprocal Willis Coupling in Zero-Index Moving Media, Phys. Rev. Lett. 123, 064301 (2019).

[32] J. Vasseur, A.-C. Hladky-Hennion, B. Djafari-Rouhani, F. Duval, B. Dubus, Y. Pennec, and P. A. Deymier, Waveguiding in two-dimensional piezoelectric phononic crystal plates, J. Appl. Phys. 101, 114904 (2007).

[33] A.-C. Hladky-Hennion and J.-N. Decarpigny, Finite element modeling of active periodic structures: Application to 1-3 piezocomposites $^{a}$, J. Acoust. Soc. Am. 94, 621 (1993).

[34] Z. Hou and B. Assouar, Tunable elastic parity-time symmetric structure based on the shunted piezoelectric materials, J. Appl. Phys. 123, 085101 (2018).

[35] Z. Hou, H. Ni, and B. Assouar, PT Symmetry for Elastic Negative Refraction, Phys. Rev. Appl. 10, 044071 (2018).

[36] Q. Wu, Y. Chen, and G. Huang, Asymmetric scattering of flexural waves in a parity-time symmetric metamaterial beam, J. Acoust. Soc. Am. 146, 850 (2019).

[37] Z. Lu and A. N. Norris, Non-reciprocal wave transmission in a bilinear spring-mass system, J. Vib. Acoust. 142, 021006 (2020).

[38] Computer code COMSOL MULTIPHYSICS, Version 5.6.

[39] Metals and alloys-densities, https://www.engineeringtoolbox. com/metal-alloys-densities-d_50.html.

[40] M. Yang, Z. Ye, M. Farhat, and P.-Y. Chen, Enhanced RadioFrequency Sensors Based on a Self-Dual Emitter-Absorber, Phys. Rev. Appl. 15, 014026 (2021).

[41] B. Yan, K. Wang, Z. Hu, C. Wu, and X. Zhang, Shunt damping vibration control technology: A review, Appl. Sci. 7, 494 (2017).

[42] Less than $10 \%$ change in the EP frequency compared to $400 \%$ for the flexural case for an equivalent change in the relative imaginary part; also for acoustics the frequency is redshifted with increasing imaginary part, while it is blueshifted in the flexural wave case presented here.

[43] S. Longhi, PT-symmetric laser absorber, Phys. Rev. A 82, 031801(R) (2010).

[44] Y. D. Chong, L. Ge, and A. D. Stone, PT-symmetry Breaking and Laser-Absorber Modes in Optical Scattering Systems, Phys. Rev. Lett. 106, 093902 (2011). 
[45] M. Farhat, M. Yang, Z. Ye, and P.-Y. Chen, PT-symmetric absorber-laser enables electromagnetic sensors with unprecedented sensitivity, ACS Photonics 7, 2080 (2020).

[46] J. Mei, G. Ma, M. Yang, Z. Yang, W. Wen, and P. Sheng, Dark acoustic metamaterials as super absorbers for low-frequency sound, Nat. Commun. 3, 1 (2012).

[47] Y. Li and B. M. Assouar, Acoustic metasurface-based perfect absorber with deep subwavelength thickness, Appl. Phys. Lett. 108, 063502 (2016).
[48] J. Song, P. Bai, Z. Hang, and Y. Lai, Acoustic coherent perfect absorbers, New J. Phys. 16, 033026 (2014).

[49] Z. Chang and G. Hu, Elastic wave omnidirectional absorbers designed by transformation method, Appl. Phys. Lett. 101, 054102 (2012).

[50] G. Trainiti, Y. Ra'di, M. Ruzzene, and A. Alù, Coherent virtual absorption of elastodynamic waves, Sci. Adv. 5, eaaw3255 (2019). 